\title{
Elastostatica di una sfera stratificata \\ e sue deformazioni causate da masse superficiali $\left(^{*}\right)$
}

\author{
Michele Caputo (**)
}

Ricevuto il 29 Luglio 1961

Il calcolo delle deformazioni di una sfera elastica soggetta a forze superficiali ed a forze di massa è di notevole importanza nella soluzione di parecchi problemi di geodesia, geofisica e geologia; ciò è stato messo in rilievo in molte pubblicazioni precedenti da Love (1911), Nishimura (1943), Takeuchi (1950), Jobert (1960), Slichter (1960) ed altri.

Il primo lavoro sull'argomento risale ormai a più di un secolo fa (1854) ed è dovuto a Lamé. La prima trattazione che unisce rigore logico a praticità di formule risale appena al 1949 ed è dovuta a G. Fichera. In questo lavoro Fichera risolve il problema dello strato sferico elastico omogeneo assoggettato a forze superficiali dotate di simmetria assiale. Seguiva immediatamente un lavoro di Aquaro che generalizzava il risultato precedente togliendo la limitazione della simmetria assiale.

I primi risultati numerici furono dati da Niskanen (1943) allo scopo di gettare luce sul problema del sollevamento della regione finnicoscandinava, ma le tabelle fornite da questo autore, per quanto notevoli se considerate in rapporto ai mezzi di calcolo allora esistenti, sono inutilizzabili per le moderne necessità.

Il recente sviluppo degli studi delle maree terrestri che vengono ora osservate in circa cento stazioni distribuite in tutto il mondo, ha riproposto l'argomento con grande attualità per il calcolo degli effetti cosiddetti secondari ed indiretti. Gli effetti indiretti sono causati da

(*) Presentato al IV Symposium sulle Maree Terrestri, Bruxelles 6-11 Giugno 1961 .

(**) Istituto di Topografia Geodesia e Geofisica, Università degli Studi di Trieste. 
forze derivanti da quelle di marea, che quindi hanno gli stessi periodi di queste ultime anche se con ampiezze relative e fasi diverse: di questo tipo sono gli effetti causati dalle acque spostate dalle maree; gli effetti secondari sono causati da forze variabili nel tempo, con periodi diversi da quelli delle maree: di questo tipo sono gli effetti causati dalle sesse.

Nelle misure delle maree terrestri, le variazioni della gravità sono ora misurate con precisione superiore a $0.1 \mu$ gal, cioè circa $0.5 \%$ dell'effetto totale $\left({ }^{*}\right)$, le deviazioni della verticale con precisione $0.05 \mathrm{~m}$ sec cioè $1 \%$ della deviazione totale (Marussi 1960). Essendo risultato dalle comunicazioni ai recenti Symposium sulle Maree Terrestri (Marussi (1960), Zadro (1961), Verbaandert, Melchior (1961), Tomaschek (1961), Bossolasco-Cicconi (1961)) che gli effetti indiretti e secondari possono talvolta raggiungere punte del $40 \%$ dell'effetto registrato, si è imposto urgentemente il problema del calcolo degli effetti medesimi.

I primi risultati numerici utili in merito sono stati dati da Slichter e dall'autore della presente nota (1960) nel caso di un modello della Terra costituito da uno strato sferico contenente un nucleo liquido ed assoggettato a forze superficiali dotate di simmetria assiale. In detta nota infatti si forniscono gli spostamenti radiali e tangenziali di grado in grado per varie distribuzioni di forze superficiali.

Nei lavori teorici e numerici precedentemente citati, non si tenne conto dell'attrazione esercitata sulla Terra dalle masse distribuite sulla sua superficie ma solo dal carico che esse esercitano sulla superficie stessa $(* *)$.

Il risultato di Fichera veniva poi ripreso (1961) dall'autore della presenta nota ed esteso alle deformazioni dotate di simmetria assiale per un modello della Terra composto da $m$ strati omogenei e da un nucleo, soggetto a forze superficiali, tenendo conto anche dell'azione di

(*) Ciò è stato ottenuto da C. L. Hager e dall'autore di questa nota con un gravimetro LaCoste Romberg in un'esperienza organizzata in collaborazione dall'Istituto di Topografia, Geodesia e Geofisica dell'Università di Trieste e dall'Institute of Geophysics dell'Università di California, in occasione dell'eclissi totale di Sole del Febbraio 1961 a Firenze.

(**) Tavole per il calcolo degli spostamenti radiali di un modello della Terra soggetto a forze derivanti da distribuzioni superficiali di masse assisimmetriche, sono state comunicate dall'autore di questa nota al XI Convegno dell'Associazione Geofisica Italiana a Roma (1961). Un metodo grafico per il calcolo degli spostamenti causati da distribuzioni generiche di masse su. perficiali è stato comunicato dall'autore di questa nota al First Western National Meeting dell'American Geophysical Union a Los Angeles (1961). 
forze di massa derivanti da una distribuzione superficiale di masse situate sulla superficie esterna della Terra.

Ma alcuni problemi di geodesia, geofisica, geologia, e fisica dei pianeti come quelli delle maree, per ovvie ragioni geografiche, necessitano che si consideri il caso più generale dei precedenti in cui sia tolta la limitazione della simmetria assiale.

Orbene il presente lavoro generalizza i risultati dei precedenti soddisfando queste necessità.

Partendo dai risultati di Aquaro, in questa nota si considera una sfera composta di $m$ strati omogenei concentrici e da un nucleo di date densità e costanti elastiche, e, assegnate che siano le forze (o gli spostamenti) sulla superficie esterna della sfera, si determinano gli sviluppi in serie che forniscono le componenti polari del vettore spostamento. Si trovano poi dette componenti anche nel caso che, oltre alle forze assegnate sulla superficie, siano presenti forze di massa originate da una distribuzione di masse sulla superficie stessa. Colle limitazioni che il loro spessore sia trascurabile rispetto al raggio della sfera e il loro potenziale sia esprimibile con una serie di funzioni armoniche sferiche. Condizioni che sono sempre soddisfatte in quei problemi di geodesia, geofisica e geologia in cui la sfera è la Terra o un pianeta e le masse superficiali sono costituite da catene montagnose, da calotte di neve e ghiaccio, o strati di acqua spostati dalle forze di marea.

1. Sia $C$ un campo elastico isotropo e omogeneo di parametri elastici $\lambda$ e $\mu$, schematizzabile nello strato sferico $T$ di centro nell'origine 0 di uno spazio cartesiano e limitato dalle due sfere $\Sigma_{1}$ e $\Sigma_{2}$ di raggi $r_{1}$ ed $r_{2}$ rispettivamente $\left(r_{1}<r_{2}, r_{1}>0\right)$.

Limitando per ora le nostre considerazioni al caso che siano assenti le forze di massa e indicando con $\mathbf{s}$ il vettore spostamento relativo ad una deformazione determinata da sole forze $f$ agenti al confrono, le condizioni necessarie e sufficienti per l'equilibrio di $C$, come è noto si scrivono

$$
\begin{aligned}
& (\lambda+2 \mu) \operatorname{grad} \operatorname{div} \mathbf{s}-\mu \operatorname{rot} \operatorname{rot} \mathbf{s}=0 \text { su } T-F T \\
& \lambda \operatorname{div} \mathbf{s} \bar{\nu}+2 \mu \frac{d \underline{s}}{d \bar{\nu}}+\mu \bar{v} \wedge \operatorname{rot} \mathbf{s}+f=0 \mathrm{su} F T
\end{aligned}
$$

essendo $\bar{\nu}$ il versore normale su $F T$ diretto verso l'esterno.

Introduciamo ora un sistema di coordinate polari $r, \hat{v}, \varphi$, col centro in 0

$$
r \geqslant 0,0 \leqslant \vartheta \leqslant \pi, 0 \leqslant \varphi<2 \pi
$$


ed indichiamo con $s_{r}, s_{\mathfrak{g}}$, $s_{\varphi}$ le componenti polari di s. In queste ipotesi vale il seguente teorema dovuto ad Aquaro:

Se $\mathbf{s}$ è un vettore verificante in $T-F T$ la [1], per ogni $r$ interno all'intervallo $\left[r_{1}, r_{2}\right]$ le sue componenti sono suscettibili dei seguenti sviluppi uniformemente convergenti in ogni insieme chiuso contenuto in $T-F T$.

$$
\begin{aligned}
& s_{r}=\sum_{0}^{\infty}{ }_{\frac{\coprod_{0}}{2 n}}^{2 n} U_{n}^{(k)}(r) Y_{n}{ }^{(k)} \\
& s_{\vartheta}=\sum_{0}^{\infty} \sum_{0}^{2 n}\left(V_{n}{ }^{(k)}(r) \frac{\partial Y_{n}^{(k)}}{\partial \vartheta}+W_{n}^{(k)}(r) \frac{\partial Y_{n}(k)}{\sin \vartheta \partial \varphi}\right) \\
& s_{\varphi}=\sum_{0}^{\infty} \sum_{0}^{2 n}\left(\frac{V_{n}^{(k)}(r)}{\sin \vartheta} \frac{\partial Y_{n}^{(k)}}{\partial \varphi}-W_{n}^{(k)}(r) \frac{\partial Y_{n}{ }^{(k)}}{\partial \vartheta}\right)
\end{aligned}
$$

ove:

$$
\begin{aligned}
& U^{(k)_{n}}=A_{\left({ }_{1}, n\right.}^{r^{n+1}}+A^{(k)_{2, n}} r^{-n}+A^{(k)_{3, n} r^{n-1}}+A^{(k)}{ }_{4, n} r^{-n-2} \\
& V^{(k)}{ }_{n}=n(n+1)\left[B^{(k)}{ }_{1, n} r^{n+1}+B^{(k)}{ }_{2, n} r^{-n}+B^{(k)}{ }_{3, n} r^{n-1}+B^{(k)}{ }_{4, n} r^{r-n-2}\right] \\
& W^{(k)}{ }_{n}=n(n+1)\left[C^{(k)}{ }_{1, n} r^{n}+C^{(k)}{ }_{2, n} r^{-n-1}\right]
\end{aligned}
$$

con

$$
\begin{aligned}
& B^{(k)}{ }_{1, n}=\frac{(n+3) \gamma+n+5}{n \gamma+n-2} \frac{A^{(k)}{ }_{1, n}}{n+1} \quad B^{(k)_{3, n}}=+\frac{A^{(k)}{ }_{3, n}}{n}, \lambda=\gamma \mu \\
& B^{(k)}{ }_{2, n}=\frac{(2-n) \gamma+4-n}{(1+n) \gamma+3+n}-\frac{A^{(k)}{ }_{2, n}}{n} \quad, \quad B^{(k)_{4, n}}=-\frac{A^{(k)_{4, n}}}{n+1}
\end{aligned}
$$

e

$$
Y_{n}^{(k)}(\vartheta, \varphi)=\left\{\begin{array}{cc}
\left(\frac{2 n+1}{4 \pi}\right)^{1 / 2} X_{n}(x) \quad, \quad k=0 \\
{\left[\frac{2 n+1}{2 \pi} \frac{(n-k) !}{(n+k) !}\right]^{1 / 2} X_{n}^{(k)}(\cos \vartheta) \sin ^{k} \vartheta \cos k \varphi, k=1,2, \ldots n} \\
{\left[\frac{2 n+1}{2 i \pi} \frac{(k-n) !}{(n+k) !}\right]^{1 / 2} X_{n}^{(k-n)}(\cos \vartheta) \sin { }^{k-n} \vartheta \sin (k-n) \varphi} \\
k=n+1, \ldots 2 n
\end{array}\right.
$$

essendo $X_{n}(\cos \vartheta)$ il polinomio di Legendre di ordine $n$, e $\mathrm{A}^{(0)}{ }_{2,0}=A^{(0)}{ }_{3,0}=0$.

Il teorema può facilmente essere esteso al caso $r_{1}=0$ trovando che

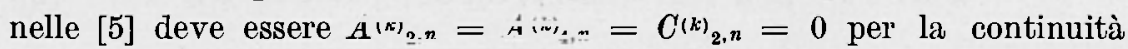
degli spostamenti nell'origine.

Le $A^{(k)}{ }_{i, n}$ sono ovviamente costanti arbitrarie e cosi le $C^{(k)}{ }_{i, n}$.

2. Abbiamo ora tutti gli elementi per applicare il metodo delle proprietà integrali alla risoluzione del primo problema esposto nell'in- 
troduzione. Consideriamo perciò una sfera costituita da $m$ strati omogenei $T_{i}$ limitati dalle sfere $\underline{\Sigma}_{i-1}, \Sigma_{i}$ di raggi $r_{i-1}$ ed $r_{i}$ rispettivamente $(i=1,2, \ldots m)$, con $r_{o}-0$ ed $r_{i-1}<r_{i}$; e siano $\lambda_{i}, \mu_{i}$ le costanti elastiche degli strati $T_{i}$. Determineremo gli spostamenti $\mathbf{s}\left(s_{r}, s_{\vartheta}, s_{\varphi}\right)$ dei punti degli strati, assegnate che siano le forze $\mathbf{f}\left(f_{r}, f_{\vartheta}, f_{\varphi}\right)$ applicate sulla superficie esterna $\bar{\Sigma}_{m}$. supposto che la sfera sia in equilibrio ed inoltre che vi sia continuità per gli spostamenti ed equilibrio per gli sforzi superficiali $P^{(i)}\left(P^{(i)} r, P^{(i)} r q, P^{(i)} r \varphi\right)$ sulle superficie $\sum_{t}(i=1 \ldots m-1)$. Dovrà perciò essere su $\sum_{m}$ :

$$
P^{(m)_{r r}}+f_{r}=0 \quad, \quad P^{(m)}{ }_{r \vartheta}+f_{\vartheta}=0 \quad, \quad P^{(m)}{ }_{r q}+f_{\varphi}=0
$$

e su $\sum_{i}(i=1 \ldots m-1)$

$$
\begin{aligned}
& s_{r}^{2}=s^{i+1}, s^{\imath} \vartheta=s^{i+1} \vartheta \quad, s^{i} \varphi=s \varphi^{i+1},
\end{aligned}
$$

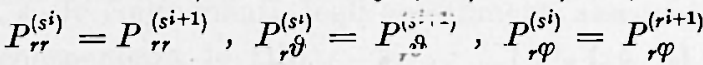

$$
\begin{aligned}
& i=1,2, \ldots, m-1, r=r_{i}
\end{aligned}
$$

essendo:

$$
\begin{aligned}
& P_{r r}=2 \mu \frac{\partial s_{r}}{\partial r}+\lambda\left[\frac{1}{r^{2}} \frac{\partial r^{2} s_{r}}{\partial r}+\frac{1}{r \sin \vartheta} \frac{\partial \sin \vartheta s_{\vartheta}}{\partial \vartheta}+\frac{1}{r \sin \vartheta} \frac{\partial s_{\varphi}}{\partial \varphi}\right] \\
& P_{r \vartheta}=\mu\left[\frac{1}{r} \frac{\partial s_{r}}{\partial \vartheta}-\because \frac{\partial s_{\vartheta} / r}{\partial r}\right] \\
& P_{r \varphi}=\mu\left[r \frac{\partial s_{\varphi} / r}{\partial r}+\frac{1}{r \sin \vartheta} \frac{\partial s_{r}}{\partial \varphi}\right]
\end{aligned}
$$

Tenuto conto che, come noto le, $Y_{n}{ }^{(k)}$ soddisfano alle relazioni

$$
\begin{gathered}
\frac{\partial}{\partial \vartheta}\left(\sin \vartheta \frac{\partial Y_{n}^{(k)}}{\partial \vartheta}\right)+-\frac{1}{\sin ^{2} \vartheta} \frac{\partial^{2} Y_{n}^{(k)}}{\partial \varphi^{2}}+n(n+1) \sin \vartheta Y_{n}^{(k)}=0 \\
{\left[Y_{n}(k)\right]_{\varphi=0}^{\varphi=2 \pi}=\left[\frac{\partial Y_{n}^{(k)}}{\partial \vartheta}\right]_{\varphi=0}^{\varphi-2 \pi}=\left[\frac{\partial Y_{n}^{(k)}}{\partial \varphi}\right]_{\varphi=0}^{\varphi=2 \pi}=0}
\end{gathered}
$$

dalle [10] con qualche integrazione per parti si ha che per ogni $r$ nell'intervallo $\left[0, r_{m}\right]$

$$
\begin{gathered}
\left.\int_{w} P_{r r}(s) Y_{n}(k) d w=2 \mu \frac{d U_{n}^{(k)}}{d r}+\lambda \mid \frac{1}{r^{2}} \frac{d\left(r^{2} U_{n}^{(k)}\right.}{d r}-\frac{n(n+1)}{r} V_{n^{(k)}}\right] \\
\int_{w}\left[P_{r \vartheta}(s) \frac{\partial Y_{n}^{(k)}}{\partial \vartheta}+P_{r \varphi} \frac{\partial Y_{n}^{(k)}}{\sin \vartheta d \varphi}\right] d w=n(n+1) \mu\left[\frac{1}{r} U_{n}^{(k)}+\right. \\
\left.+r \frac{d V_{n}^{(k) / r}}{d r}\right] \\
\int_{w}\left[P_{r \vartheta}(s) \frac{\partial Y_{n}^{(k)}}{\sin \vartheta \partial \varphi}-P_{r \varphi} \frac{\partial Y_{n}^{(k)}}{\partial \vartheta}\right] d w=n(n+1) \mu r \frac{d}{d r}\left(\frac{W_{n}^{(k)}}{r}\right)
\end{gathered}
$$


essendo

$$
\int_{w} f d w=\left.\int_{0}^{\pi} d \vartheta\right|_{\varphi} ^{2 \pi} f \sin \vartheta d \vartheta
$$

Tenuto conto della [8] e [9] si ha cosi per le condizioni di equilibrio [2] su $\Sigma_{m}$

$$
\begin{gathered}
z \mu_{m} \frac{d D_{n, m}^{(k)}}{a r}+\lambda_{m}\left(\frac{1}{r^{2} m} \frac{d\left(r^{2} U_{n, m}^{(k)}\right)}{d r}-\frac{n(n+1}{r_{m}} \bar{V}_{n, m}^{(b)}\right)= \\
=-\int_{w} f_{r} Y_{n}^{(k)} d w \\
n(n+1)\left[\frac{1}{r_{m}} \mu_{m} U_{n, m}^{(k)}+r_{m} \mu_{n} \frac{d V_{n, m}^{(k)} / r}{d r}\right]=-\int\left[f_{\vartheta} \frac{\partial Y_{n}(k)}{\partial a}+\right.
\end{gathered}
$$$$
\left.+\frac{f_{\varphi}}{\sin \vartheta} \frac{\partial Y_{n}^{(k)}}{\partial \vartheta}\right] d w
$$

$\left.n(n+1) \mu_{m} r_{m} \frac{d}{d r}-\left(\frac{m_{n, m}^{(k)}}{r}\right)=-\int\left[\frac{f_{\vartheta}}{\sin \vartheta^{\eta}} \frac{\partial Y_{n}^{(k)}}{\partial \vartheta}-t_{\varphi} \frac{\partial Y_{n}^{(k)}}{\partial \vartheta}\right] d w\right)$

ed inoltre per le condizioni di continuità di s e $\mathbf{P}$ sulle superficie $\Sigma_{i}(i-1 \ldots m-1)$ (col simbolo []$_{+}$si indica la differenza dei valori assunti dalla quantità fra parentesi negli strati $T_{i}$ e $T_{i+1}$ per $r-r_{i}$ )

$\left[2 \mu_{i} \frac{d U_{n, i}^{(k)}}{d r}+\lambda_{i}\left(\frac{1}{r_{i}^{2}} \frac{d\left(r^{2} U_{n, i}^{(k)}\right)}{d r}-\frac{n(n+1)}{r^{i}} V_{n, i}^{(k)}\right)\right]_{+}^{-}=0$

$\left[\mu_{i}\left(\frac{1}{r_{i}} \prod_{-,, i}^{(k)}+\cdots, \frac{d V_{n, i}^{(k)} / r}{d r}\right)\right]_{+}^{-}=0$

$\left[\mu_{i} \cdot r_{i}-\frac{d}{d r}\left(\frac{W_{n, i}^{(k)}}{r}\right)\right]_{+}^{-}=0$

$U_{n, i}^{(k)}=Z_{n, i+1}^{(k)}, T_{n, i}^{(k)}-T_{n, i+1}^{(k)}, T_{n, i}^{(k)}-W_{n, i+1}^{(k)}, r=i$

ove:

$U_{n, i}^{(k)}=A_{n, i}^{(k)} r^{n+1}+A_{2 n, i}^{k)} r^{-n}+A_{3 . n, i}^{(\cdots)} r^{n-1}+A_{4, n, i}^{(k)} r^{-n-2}$

$V_{n, i}^{(k)}=\left(B_{1, n, i}^{(k)} r^{n+1}+B_{2, n, i}^{(k)} r^{-n}+A_{3 n, i}^{(k)} r^{n-1}+A_{4, n, i}^{(k)} r^{-n-2}\right) n(n+1)$

$W_{n, i}^{(k)}=\left(C_{1, n, i}^{(k)} r^{n}+C_{2 . n . i}^{i \cdots \prime} r^{-n-1}\right) n(n+1)$

sono la generalizzazione delle [4] per lo strato $T_{i}$. 
Per ogni $n, k$ le equazioni [13] e [14] danno un sistema di $6 m-3$ equazioni lineari nelle $6 m-3$ incognite $A_{j, n, i}^{(k)}, C_{j, n, i}^{(k)}$. Le soluzioni del sistema determinano univocamente le [15] e di conseguenza le [4] che forniscono la risoluzione del primo problema proposto con le assegnate condizioni al contorno.

3. E ovvio che analoga trattazione può essere fatta nel caso che su $\sum_{m}$ siano assegnati gli spostamenti invece delle forze. In tal caso infatti al posto delle [8] dovremo scrivere:

$$
s_{r}^{m}=s_{r} \quad, \quad s_{\vartheta}=s_{\vartheta} \quad, \quad s \varphi^{m}=s \varphi
$$

essendo $s_{r}, s_{9}, s_{T}$ le componenti degli spostamenti assegnati su $\bar{\Sigma}_{m}$ e modificando di conseguenza le [13].

4. Veniamo ora alla risoluzione del secondo problema proposto, cioè del calcolo delle deformazioni elastiche della nostra sfera nel caso in cui oltre a forze agenti sulla superficie $\bar{\zeta}_{m}$ siano presenti anche forze di massa derivanti dall'attrazione gravitazionale di uno strato di materia di densità $d(\vartheta, \varphi)$ ed altezza $H(\vartheta, \varphi)$ distribuito su $\sum_{m}$. Se $H(\vartheta, \varphi)$ è trascurabile rispetto a $r_{m}$ allora nel primo membro di [1] si aggiunge un vettore $F$ che rappresenta una forza derivante dal potenziale:

$$
\begin{aligned}
& V=+r_{m}^{2} G \int_{0}^{\pi} d \vartheta^{\prime} \int_{0}^{2 \pi} \frac{d\left(\vartheta^{\prime}, \varphi^{\prime}\right) \mathrm{H}\left(\vartheta^{\prime}, \phi^{\prime}\right) \sin \vartheta^{\prime}}{\left[r^{2}+r_{m}^{2}-2 r r_{m} \cos \gamma\right]^{1 / 2}} d \varphi^{\prime} \\
& \cos \gamma=\cos \vartheta \cos \vartheta^{\prime}+\sin \vartheta \sin \ddot{\vartheta^{\prime}} \cos \left(\varphi-\varphi^{\prime}\right)
\end{aligned}
$$

ove $\ddot{v}$ e $\varphi^{\prime}$ sono le coordinate geografiche dei punti di $\rangle_{\prime_{m}}$ e $G$ la costante d'attrazione universale.

Se il prodotto $d(\vartheta, \varphi) H(\vartheta, \varphi)$ è dato da una serie di funzioni armoniche sferiche $\sum_{0}^{\infty}{ }_{n} \sum_{0}^{2 n}{ }_{0} A_{n, k} Y^{(k)}{ }_{n}(\vartheta, \varphi)$ allora per $V$ si ha notoriamente l'espressione:

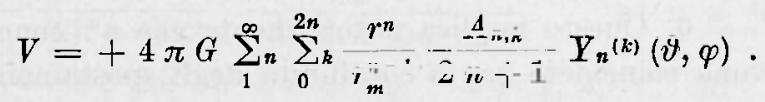


Essendo ora $V$ espressa da una serie di funzioni armoniche sferiche si verifica che un integrale particolare della [1] è dato da

$$
\begin{aligned}
& \bar{s}_{r}{ }^{i}=-\sum_{0}^{\infty} \sum_{0}^{2 n} \frac{2(n+2) G \pi \lambda_{i} A_{1, k} r^{n+1}}{(2 n+1)(2 n+3)\left(\lambda_{i}+2 \mu_{i}\right) r_{m}^{n-1}} Y_{n}{ }^{(k)} \\
& \bar{s}_{\vartheta}{ }_{\vartheta}=-\sum_{0}^{\infty} n \sum_{0}^{2 n} \frac{2 G \pi d_{i} A_{n \cdot}{ }^{k} r^{n+1}}{(2 n+1)(2 n+3)\left(\lambda_{i}+2 \mu_{i}\right) r_{m}^{n-1}} \frac{\partial Y_{n}{ }^{(k)}}{\partial \vartheta} \\
& \bar{s}_{\varphi}=-\sum_{0}^{\infty} n \sum_{v}^{2 n} \frac{2 G \pi d_{i} A_{n, k} r^{n+1}}{(2 n+1)(2 n+3)\left(\lambda_{i}+2 \mu_{i}\right) r_{n}^{n-1}} \frac{\partial Y_{n}{ }^{(k)}}{\sin \vartheta \partial \varphi} \\
& s^{i} \varphi=s_{\vartheta}^{i}=s_{r}^{i}=0 \quad, \text { se } n=k=0
\end{aligned}
$$

per $r_{i} \leqslant r \leqslant r_{i+1}$, essendo $d_{i}$ la densità dello strato sferico $T_{i}$. L'integrale generale $\mathbf{S}_{\mathfrak{\imath}}$ della [1] con le forze di massa $F$ è quindi dato da:

$$
S_{r}^{i}=s_{r}^{i}+\bar{s}_{r}^{i}, S_{\vartheta}{ }_{\vartheta}=s^{i} \vartheta+\bar{s}_{\vartheta}^{i}, S^{i} \varphi=s^{i} \varphi+\bar{s}^{i} \varphi
$$

Per soddisfare le condizioni al contorno su $\zeta_{m}$, e di continuità per gli spostamenti e gli sforzi su $\Sigma_{i}(i=1 \ldots m-1)$, ci si serve ancora delle [8] e [9] dove si sostituisce $s^{i}+s^{i}$ al posto di $s^{i}$. Si ottengono cosi $6 m-3$ equazioni analoghe alle [13] e [14], che si ritiene inutile scrivere, e che determinano le $6 m-3$ costanti $A_{i n i}^{i n} C_{i n, i}^{i n j}$ che sostituite nelle [15] colle [4], [19] e [20] danno la soluzione del problema proposto.

5. Per provare l'unicità della soluzione in entrambi i problemi considerati osserviamo che, indicando con $s^{*_{i}}\left(s^{*_{i}}, s_{\vartheta^{*}}, s \varphi^{*_{i}}\right)$ la differenza di due eventuali soluzioni distinte e con $\mathrm{p}^{* i}\left(p_{v_{1}}^{* i}, p_{\vartheta}^{* i}, p_{\varphi}^{* i}\right)$ la differenza degli sforzi associati, notoriamente si ha per ogni strato $T_{i}$

$$
\int_{\Sigma_{i}} \mathbf{P} *_{i} \times \mathbf{s}^{*_{i}} d \Sigma_{i}-\int_{\Sigma_{r_{-1}}} \mathbf{P} *_{i-1} \times \mathbf{s}^{*_{i-1}} d \Sigma_{i-1}=2 \int_{T_{i}} W_{i} d T_{i}
$$

essendo $W_{i}$ il potenziale elastico: sommando quindi rispetto ad $i$

$$
\int_{\Sigma_{m}} \mathbf{P}^{* m} \times \mathbf{s}^{* m} d \Sigma_{m}=2 \sum_{\sum_{i}}^{m} \int_{T_{i}} \mid W_{i} d T_{t}
$$

Siccome su $\bar{\zeta}_{n}$ si ha $\mathrm{p}^{* m}=0$ nel caso degli assegnati sforzi, ovvero $\mathbf{s}^{*_{m}}=0$ nel caso degli assegnati spostamenti, segue in tutti $\mathrm{i}$ casi

$$
\sum_{1}^{m} \int_{T_{i}} W_{i} d T_{i}=0
$$

e perciò $W_{t}=0$. Questo implica notoriamente che $\mathbf{s}^{* i}$ sono traslazioni le quali devono coincidere per la continuità degli spostamenti sulle $\sum i$. 
Nel caso degli assegnati spostamenti in particolare questa traslazione è nulla poiché $\mathrm{s}^{* m}=0$.

6. Un caso di notevole significato è quello in cui le forze applicate su $\Sigma_{m}$ sono date dallo strato di materia di densità $d(\vartheta, \varphi)$ e altezza $H(\vartheta, \varphi)$ adagiato su $\bar{\gamma}_{m}$ come definito al n. 4 .

Le forze applicate su $\sum_{m}$ siano perciò date da $f_{r}=g d(\vartheta, \varphi) H(\vartheta, \varphi)=g \sum_{0}^{n} \sum_{0}^{2 n} A_{n, k} Y_{n}^{(k)}(\vartheta, \varphi) \quad, \quad f_{\vartheta}=f_{m}=0$

$g=\frac{4}{3} \pi G \sum_{i}^{m} \frac{\left(r^{3} i-r^{3}{ }_{i-1}\right) d_{i}}{r_{m}}$

essendo $g$ la gravità su $\sum_{m}$.

Il sistema di condizioni al contorno si scrive per la condizione di equilibrio su $\sum_{m}$

$$
\begin{aligned}
& P_{r r}\left(\mathbf{S}^{m}\right)=P_{r r}\left(\mathbf{s}^{m}+\overline{\mathbf{s}}^{m}\right)=-g \sum_{0}^{m} \sum_{0}^{2 n} A_{n, k} Y_{n}(k)(\vartheta, \varphi) \\
& P_{r \vartheta}\left(\mathbf{S}^{m}\right)=P_{r \vartheta}\left(\mathbf{S}^{m}+\overline{\mathbf{s}}^{m}\right)=0 \\
& P_{r \varphi}\left(\mathbf{S}^{m}\right)=P_{r \varphi}\left(\mathbf{S}^{m}+\overline{\mathbf{S}}^{m}\right)=0 \quad, \quad r=r_{m} .
\end{aligned}
$$

e per la condizione di continuità degli spostamenti e sforzi su $\sum_{i}(i=1,2, \ldots, m-1)$

$$
\begin{array}{ll}
{\left[s_{r}^{i}+\bar{s},\right]_{+}^{-}=0,} & {\left[P_{r r}^{i}(\mathbf{S})\right]_{+}^{-}=0} \\
{\left[s_{\vartheta}^{i}+\bar{s}_{\vartheta}^{i}\right]_{+}^{-}=0,} & {\left[P_{r \vartheta}^{i}(\mathbf{S})\right]_{+}^{-}=0} \\
{\left[s_{\varphi}^{i}+\bar{s}_{\varphi}^{i}\right]_{+}^{-}=0,} & {\left[P_{r \varphi}^{i}(\mathbf{S})\right]_{+}^{-}=0 \quad r=r}
\end{array}
$$

L'integrale generale $\mathbf{S} i$ colle forze di massa $\mathbf{F}\left(F_{r}, F_{\vartheta}, F_{\varphi}\right)$ derivate dal potenziale $V$ dato dalla [17], tenuto conto della [19], si scrive:

$$
\begin{aligned}
S_{r}{ }^{\imath}= & \sum_{0}^{\infty} n \sum_{0}^{2 n}\left[U_{n, i}^{(k)}+Q_{i}(n+2) r^{n+1}\right] Y_{n}^{(k)} \\
S_{\vartheta}^{i}= & \sum_{0}^{\infty} n \sum_{0}^{2 n}\left[V_{n, i}^{(k)}+Q_{i} r^{n+1}\right] \frac{\partial Y_{n}^{(k)}}{\partial \vartheta}+W_{n, i}^{(k)} \frac{1}{\sin \vartheta} \frac{\partial Y_{n}^{(k)}}{\partial \varphi} \\
S_{\varphi}^{i}= & \sum_{0}^{\infty} \sum_{0}^{2 n}\left[V_{n, i}^{(k)}+Q_{i} r^{n+1}\right] \frac{1}{\sin \vartheta} \frac{\partial Y_{n}^{(k)}}{\partial \varphi}-\eta_{n, i}^{(k)} \frac{\partial Y_{n}^{(k)}}{\partial \vartheta} \\
Q_{i}= & -\frac{2 \pi G d_{i} A_{n, k}}{(2 n+1)(2 n+3)\left(\lambda_{i}+2 \mu_{i}\right) r_{m}{ }^{n-1}} \quad \text { se } n \neq 0 \\
& Q_{i}=0, \text { se } k=n=0, \quad i=1,2, \ldots . m .
\end{aligned}
$$


Perciò applicando il procedimento del n. 2, il sistema delle condizioni al contorno diventa, su $\sum_{m}$ :

$$
\begin{aligned}
& 2 \mu_{n} \frac{d U_{n, m}^{(k)}}{d r}+\lambda_{m}\left(\frac{1}{r^{2}} \frac{d r^{i} U_{n_{1} m}^{(k)}}{d r}-\frac{n(n+1)}{r} V_{n, m}^{(k)}\right)^{\prime}- \\
& =-2 \mu_{m}(n+1)(n+2) Q_{m} r_{m^{n}}-\lambda_{m} Q_{m} 2(2 n+3) r^{n}-\mid f_{r} Y_{n}^{k} d w \\
& \frac{\bar{U}_{m, n}^{(k)}}{r}+r \frac{d V_{m, n}^{\prime)} / r}{d r}=-2(n+1) Q_{m} r^{n} \\
& \frac{d}{d r} \frac{W_{m, u}^{(k)}}{r}=0 \quad, \quad r=r_{m}
\end{aligned}
$$

e su $\Sigma_{i}$

$$
\begin{aligned}
& {\left[2 \mu_{i} \frac{d U_{n, i}^{(k)}}{d r}+\lambda_{i}\left(\frac{1}{r^{2}} \frac{d r^{2} U_{n, i}^{(k)}}{d r}-\frac{n(n+1) V_{n, i}^{(k)}}{r}\right)+\right.} \\
& \left.\left.+\left[2 \mu_{i}(n+1)(n+2)+\lambda_{i} 2(2 n+3)\right] r^{n} Q_{i}\right]\right]_{+}^{-}=0 \\
& {\left[\mu_{i}\left[\frac{U_{n, i}^{(k)}}{r}+r \cdot \frac{d V_{t, i}^{(k)} / r}{d r}+2(n+1) r^{n} Q_{i}\right]\right]_{+}^{-}=0} \\
& {\left[\mu_{i} \frac{d}{d r} \frac{W_{n, i}^{(k)}}{r}\right]_{+}^{-}=0} \\
& {\left[U_{n, i}^{(k)}+(n+2) Q_{i} r^{n+1}\right]_{+}=0} \\
& {\left[V_{n, i}^{(k)}+Q_{i} r^{n+1}\right]_{+}^{-}=0} \\
& {\left[W_{n, i}^{(k)}\right]_{+}=0 \quad, \quad r=r_{i} \quad, \quad i=1,2, \ldots . m-1}
\end{aligned}
$$

7. Per il calcolo degli effetti secondari ed indiretti nello studio delle maree terrestri è sufficiente assumere un modello della Terra costituito da un mantello contenente un nucleo liquido $\left(\mu_{1}=0\right)$. In tale caso inoltre le forze di deformazione sono del tipo di quelle assunte nel numero precedente. Per il calcolo degli spostamenti si procede come segue. Nelle formule [24], [25], [28], in cui le lettere affette da indice $m$ indicano quantità che si riferiscono al mantello, $m$ diventa uguale a 2 ; nella [27] l'indice $i$ assume successivamente $\mathrm{i}$ valori 1 e 2, e nelle [26], [29] il valore 1 . 
Inoltre come già detto si assume $\mu_{1}=0$ essendo il nucleo liquido. In seguito a quest'ultima ipotesi si assume anche che gli spostamenti e gli sforzi non radiali siano nulli nel nucleo, per cui

$$
P_{r, \vartheta}^{1}=P_{r, \varphi}^{1}=0
$$

ed inoltre su $\Sigma_{1}$

$$
P_{r r}^{2}=P_{r}^{1} \quad, \quad P_{r \vartheta}^{2}=P_{r}^{1}{ }_{r}=0, \quad r-r_{1}
$$

Dei sistemi [28] e [29] delle condizioni al contorno il primo non subisce trasformazione (solo si pone $m=2$ ), il secondo invece si scrive per le [30] e [31]:

$$
\begin{aligned}
& 2 \mu_{2} \frac{d U_{n, 2}^{(k)}}{d r}+\lambda_{-}\left(\frac{1}{r^{2}} \frac{d r^{2} U_{n, 2}^{(k)}}{d r} \frac{n(n+1)}{r} r_{n, 2}^{(k)}\right)+ \\
& \quad+2 Q_{2} r^{n}\left[(2 n+3) \lambda_{2}+(n+1)(n+2) \mu_{2}\right]= \\
& \quad=\lambda_{1}\left[\frac{1}{r^{2}} \frac{d r^{2} U_{n, 1}^{(k)}}{d r}-\frac{n(n+1)}{r} V_{n, 1}^{(k)}+2 Q_{1} r^{n}(2 n+3)\right] \\
& \frac{U_{n, 2}^{(k)}}{r}+r \frac{d V_{n, 2}^{(k)} / r}{d r}+2(n+1) Q_{2} r^{n}=0 \\
& \frac{d}{d r} \frac{W_{n, 2}^{(k)}}{r}=0 \\
& U_{n, 2}^{(k)}+(n+2) r^{n+1} Q_{2}=U_{n, 1}^{(w)}+(n+2) Q_{1} r^{n+1} \\
& V_{n, 2}^{(k)}+Q_{2} r^{n+1}=V_{n, 1}^{(i)}+Q_{1} r^{n+1} \\
& W_{n,}^{(k)}=W_{n, 1}^{(k)} \quad, \quad r=r_{1}
\end{aligned}
$$

8. Per il calcolo delle soluzioni del sistema delle [28] (con $m=2$ ) e [32] è opportuno fare le seguenti ulteriori considerazioni.

La terza delle [28] e la terza e sesta delle [32] formano un sistema di tre equazioni lineari omogenee nelle tre incognite $C_{1,2}^{(k)}, C_{2, \pi, 2}^{k)}$, $C_{1, n, 1}^{(k)}$, con determinante non nullo (tranne che per $n=1$ ) per cui si ha:

$$
C_{1, n, 1}^{(k)}=\sigma_{2, n, 1}^{(k)}=G_{1, n, 1}^{(k)}=0
$$


Le rimanenti equazioni delle [28] e [32]

$$
\begin{aligned}
& 2 \mu_{2} \frac{d U_{n, 2}^{(k)}}{d r}+\lambda_{2}\left(\frac{1}{r^{2}} \frac{d r^{2} U_{n, 2}^{(k)}}{d r}-\frac{n(n+1)}{r} V_{n, 2}^{(k)}\right)= \\
& =-2 \mu_{2}(n+1)(n+2) Q_{2} r^{n}-\lambda_{2} Q_{2} 2(2 n+3) r^{n}-\int_{n} f_{r} Y_{n}(k) d w
\end{aligned}
$$

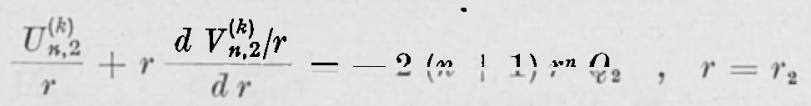

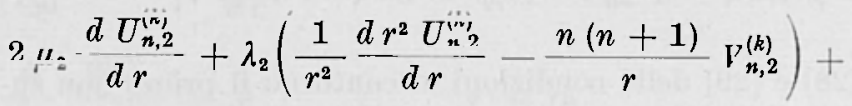

$$
\begin{aligned}
& +2 Q_{2} r^{n}\left[(n+1)(n+2) \mu_{2}+(2 n+3) \lambda_{2}\right]= \\
& =\lambda_{1}\left[\frac{1}{r^{2}} \frac{d U_{n 1}^{(k)}}{d r}-\frac{n(n+1)}{r} V_{n, 1}^{(b)}+2 Q_{1} r^{n}(2 n+3)\right] \\
& \frac{U_{n 2}^{(k)}}{r}+r \frac{d V_{, 2}^{(k)} / r}{d r}+2(n+1) Q_{2} r^{n}=0 \\
& U_{n,}^{(k)}+(n+2) Q_{2}, \ldots:=U_{n_{1}}^{(\ldots)}+(n+2) Q_{1} r^{n+1} \\
& V_{n, 2}^{(k)}+Q_{2} r^{n+1}=V_{n, 1}^{(\xi)}+Q_{1} r^{n+1}, r=r_{1}
\end{aligned}
$$

per ogni $n$ e $k$ danno incognite $A_{1, n, 2}^{(k)}, A_{2, n, 2}^{(k)}, A_{3, n, 2}^{(k)}, A_{4, n, 2}^{(k)}, A_{1, n 1}^{(k)}$, $A_{3 n, 1}^{(k)}$, in particolare per $n=k=0$ il sistema

$$
\begin{aligned}
& \left(\lambda_{2}+2 \mu_{2}\right) \frac{d U_{, 2}^{(o)}}{d r}+2 \lambda_{2} \frac{U_{0,2}^{(o)}}{r}-\lambda_{1}\left(\frac{d U_{\because,:}^{(o)}}{d r}+2 \frac{\Pi_{-0.1}^{(o)}}{r}\right) \\
& U_{o 2}^{k}=ت_{o, 1}^{(o)}, r=r_{1} \\
& \left(\lambda_{2}+2 \mu_{2}\right) \frac{\lambda T_{w}^{(o)}}{i r}+2 \lambda_{2} \cdot \frac{U_{o, 2}^{(k)}}{r}=-\int_{w} f_{r} d w \quad, \quad r=r_{2}
\end{aligned}
$$

fornisce $A_{1,0,2}^{(0)} \quad, \quad A_{40,2}^{(0)}, A_{10,1}^{(0)}$.

9. Una speciale semplificazione si ottiene supponendo che la pressione nel nucleo sia costante

$$
P^{1_{r r}}=\text { cost. }=P
$$

Dall'integrale

$$
\int_{w} P_{r r} Y_{n}(k) d w= \begin{cases}2 P \sqrt{\pi} & \text { se } n=k=0 \\ 0 & \text { se } k \neq 0\end{cases}
$$


per le [11], segue che per ogni $r$ deve essere

$\lambda_{1}\left[\frac{1}{r^{2}} \frac{d r^{2} U_{o 1}^{(0)}}{d r}\right]=2 P V \bar{\pi} \quad$ se $n=k=0$
$\lambda_{1}\left[\frac{1}{r^{2}} \frac{d r^{2} U_{n, 1}^{(\kappa)}}{d r}-\frac{n(n+1)}{r} V_{n, 1}^{(k)}+2(2 n+3) Q_{1} r^{n}\right]=0$ se $k \neq 0$

ovvero

$3 \lambda_{1} A_{1,0,1}^{(0)}=2 P / \bar{\pi}$

$\left[\mathrm{n}+3-n k_{1}\right] A_{1, n, 1}^{(k)}+2(2 n+3) Q_{1}=0, k_{1}=\frac{(n+3) \gamma+n+5}{n \gamma+n-2}$

ed infine:

$$
\begin{aligned}
& A_{1,0,1}^{(o)}-\frac{2 P V^{\prime}}{3 \lambda_{1}} \\
& A_{1, n, 1}^{(k)}=-\frac{2(2 n+3) Q_{1}}{n+3-n k_{1}}
\end{aligned}
$$

Pertanto i sistemi [34] e [35] si scrivono rispettivamente

$$
\begin{aligned}
& 2_{\mu, 2} \frac{d U_{n, 2}^{(k)}}{d r}+\lambda_{2}\left(\frac{1}{r^{2}} \frac{d r^{2} U_{n, 2}^{(k)}}{d r}-\frac{n(n+1)}{r} V_{n, 2}^{(k)}\right)= \\
& =-2 Q_{2} r^{n}\left[(2 n+3) \lambda_{2}+(n+1)(n+2) \mu_{2}\right]-\int_{w} f_{r} Y_{n}{ }^{(k)} d w \\
& \frac{U_{n, 2}^{(k)}}{r}+r \frac{d V_{n, 2}^{(k)} / r}{d r}+2(n+1) Q_{2} r^{n}=0 \quad, \quad r=r_{2} \\
& 2 \mu_{2} \frac{d D_{n, 2}^{(k)}}{d r}+\lambda_{2}\left(\begin{array}{c}
1 \\
r^{2}
\end{array} \frac{d r^{2} U_{n, 2}^{(i)}}{d r}-\frac{n(n+1)}{r} V_{n, 2}^{(k)}\right)+ \\
& +2 Q_{2} r^{n}\left[(2 n+3) \lambda_{2}+(n+2)(n+1) \mu_{2}\right]=0
\end{aligned}
$$

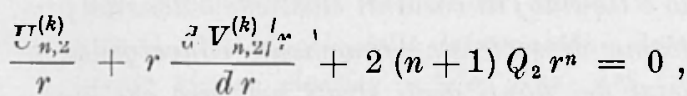

$$
\begin{aligned}
& U_{n, 2}^{(k)}+(n+2) Q_{2} r^{n-1}=U_{n .1}^{(m)}+(n+2) Q_{1} r^{n+1}, \\
& A_{1, n, 1}^{(k)}=-\frac{2(2 n+3) Q_{1}}{n+3-n(n+1) k_{1}} \quad, \quad r=r_{1}
\end{aligned}
$$


e

$$
\begin{aligned}
& \left(\lambda_{2}+2 \mu_{2}\right) \frac{d T_{-v,-}^{(o)}}{d r}+2 \lambda_{2} \frac{T_{\mathrm{v}, \mathrm{L}}^{(o)}}{r}=-\int_{w} f_{r} d w \quad, \quad r=r_{2} \\
& \left.\begin{array}{c}
\left(\lambda_{2}+2 \mu_{2}\right) \frac{\frac{d T_{-, \mathrm{L}}^{(o)}}{d r}}{d r}+2 \lambda_{2} \frac{D_{0,2}^{(o)}}{r}=3 \lambda_{1} \dot{A}_{1,0,1}^{(o)}=\left.2 P\right|^{\prime} \pi \\
U_{0,2}^{(o)}=A_{1,0,1}^{(0)} \quad, \quad r=r_{1} .
\end{array}\right\}
\end{aligned}
$$

Le costanti $A_{i, n, 2}^{(b)}, A_{i, n, 1}^{(b)}$ che si determinano colle [41] e [42] sostituite nella [15] colle [6] e [27] danno le soluzioni del problema.

A queste semplificazioni si può pervenire anche con considerazioni di carattere analitico più rigorose. Si è preferito procedere come sopra per semplicità di forma e per restare in armonia col metodo seguito precedentemente.

E opportuno osservare che se interessano solo gli spostamenti per il mantello, le corrispondenti $A_{i, n, 2}^{(k)}$ sono fornite dalle prime quattro equazioni del sistema [41] e dalle [42]; tali spostamenti si scrivono

$$
\begin{aligned}
& S_{r}^{2}=\sum_{0}^{\infty} n \sum_{0}^{2 n}\left(U_{n, 2}^{(k)}+Q_{2}(n+2) r^{n+1}\right) Y_{n}^{(k)} \\
& S_{\vartheta^{2}}=\sum_{0}^{n} \sum_{0}^{2 n}\left(V_{n, 2}^{(k)}+Q_{2} r^{n+1}\right) \frac{\partial Y_{n}^{k}}{\partial i n} \\
& S \varphi^{2}=\sum_{0}^{\infty} \sum_{0}^{2 n} \sum_{k}\left(V_{n, 2}^{(k)}+Q_{2} r^{n+1}\right) \frac{1}{\sin \vartheta} \frac{\partial Y_{n}^{(k)}}{\partial \varphi} \text {. }
\end{aligned}
$$

10. Quanto sviluppato nei numeri 7, 8 e 9 per lo studio delle maree terrestri, vale anche per lo studio delle maree di un pianeta, compreso il caso che questo sia privo di nucleo liquido ed interamente solido, in cui si deve porre $r_{i}=0$, nelle formule, con particolari immediate semplificazioni su cui non è necessario soffermarsi.

\section{RIASSUNTO}

Si considera una sfera elastica costituita da $m-1$ strati sferici concentrici contenenti un nucleo (solido o liquido) di costanti elastiche e densità prefissate. Si determinano sotto forma di serie uniformemente convergenti, le formule che danno gli spostamenti dei punti degli strati nel caso che siano assegnate le forze (o gli spostamenti) sulla superficie esterna e nel caso che siano presenti anche forze di massa derivanti da una distribuzione superficiale di masse. Si fa applicazione dei risultati ottenuti al calcolo degli effetti secondari e indiretti nello studio delle maree terrestri. 


\section{$S U M M A R Y$}

Series expressions are developped for the surface displacements of a spherical model deformed by surface traction and by body forces arising from a distribution of mass over its surface. The model consists of $m-1$ homogeneous alastic spherical shells plus an inner core which is treated either as elastic or as liquid. The computation of the indirect and secondary effects in the study of the Earth tides is then treated.

\section{RIFERIMENTI}

Almansi, Sulla deformazione della sfera isotropa. "Memorie Accademia Torino ", (1897).

Aquaro G., Sul calcolo delle deformazioni di uno strato sferico elastico. "Acc. Naz. dei Lincei - Classe Scienze Fis. Mat. e Nat. ", Serie VIII, 7, 6, 289-297, (1949).

Bossolasco M., Cicconi G., Sur les Variations du vecteur gravite à Gênes. "Quatrième Symposium International sur les marées terrestres, Communications de l'Obs. R. de Belgique", 188, 109-121, (1961).

Caputo M., a) Deformation of a layered spherical Earth by an axially simmetric surface mass distribution. "J. Geophys. Research ", 5, 479.485, (1961).

- b) Deformazioni di un modello della terra causate da distribuzioni superficiali di masse gravitazionali. "Atti X Convegno dell'Associazione Geofisica Italiana ", 157-165, Roma 1960.

- c) Elastodinamica ed elastostatica di un modello della Terra e sue autooscillazioni toroidali "Boll. di Geof. Teorica ed Applicata ", 1-20, III, 10, (1961).

- d) Tabelle delle deformazioni di un modello della Terra causate da masse gravitazionali assisimmetriche. "Atti XI Convegno dell'Associazione Geofisica Italiana ", Roma, Novembre 1961.

- $\theta$ ) Tables of the deformations of an Earth model by surface mass distributions. "First National Western Meeting of American Geophysical Union", Los Angeles Calif. December 1961.

Fichera G., Sul calcolo delle deformazioni, dotate di simmetria assiale, di uno strato sferico elastico. "Acc. Naz. dei Lincei, Classe Scienze Fis. Mat. e Nat. ", Serie VIII, 6, 5, 582-589, (1949).

Hовsоn E. W., The Theory of spherical and ellipsoidal harmonics. Cambridge at the University Press, (1931).

Jobert G., Perturbation des marées terrestres. "Annales de Géophysique ", 16, 1, 1.55, (1960). 
LАмt, Mémoire sur l'équilibre d'élasticité des enveloppes sphériques. "Journal de Liouville ", (1854).

Love A. E. H., Some problems of geodynamics. Cambridge at the University Press, (1911).

- The mathematical theory of elasticity. Dover Edition, 252-254, (1944).

Marcolongo, Sulla deformazione della sfera isotropa "Annali di Matematica ", (1895).

Marussi A., I primi risultati ottenuti nella Stazione per lo Studio delle Maree della verticale della Grotta Gigante. "Bollettino di Geodesia e Scienze Affini ", XIX, 4, 645-667, (1960). (Numero speciale).

Niskanen E., On the deformation of the earth's orust under the weight of a glacial ice-load and related phenomena. "Publications Isostatic Institute ", 12, (Helsinki 1943).

Sliciter L. B., Caputo M., Deformation of an earth model by surface pressures. "Journal of Geophysical Research ", 12, 4151-4157, (1960).

TAKEUCHI H., On earth tide of the compressible earth of variable density and elasticity. "Transaction American Geophysical Union ", 31, 5, 651-689, (1950).

Tomaschek R., Non-elastic tilt of the Earth's crust due to meteorological pressure distribution. "Geofisica Pura ed Applicata ", 25, 17-25, (1953).

VerbaAndert J., MeLchior P., Les stations géophysiques souterraines et les pendules horizontaux de l'Observatoire Royal de Belgique. "Quatrième Symposium International sur les marées terrestres, Communication de l'Obs. R. de Belgique ", 188, 192-201, (1961).

ZADro M., L'activité de la Station de Trieste (Grotta Gigante) pendant la periode de Juin 1960, à Mars 1961. "Quatrième Symposium International sur les marées terrestres. Communications de l'Obs. R. de Belgiquen, 188, 165-168, (1961). 